\title{
JEROME SEYMOUR BRUNER: \\ DE LA PERCEPCIÓN AL LENGUAJE
}

Mikel Aramburu Oyarbide

Facultad de Psicología, Universidad del País Vasco, España

\section{LA PERCEPCIÓN}

Bruner hizo un gran esfuerzo por demostrar la influencia que tienen las variables cognitivas y motivacionales en la percepción. Desde este punto de vista, distingue tres fases en la percepción: 1) Una fase pre-perceptiva, en la que el sujeto está a la expectativa de un determinado acontecimiento, llevado por sus esquemas intelectuales o motivacionales. 2) La fase de la recepción de la información. 3) La fase de evaluación de las hipótesis perceptivas, en la que el sujeto juzga la adecuación existente entre sus expectativas anteriores y la información recibida. Si las hipótesis se confirman, estamos en presencia de un nuevo percepto. Si no se confirman, se formulan nuevas hipótesis. Algunas veces, si los objetos percibidos no se corresponden con las expectativas del sujeto, pueden darse distorsiones perceptivas, y se sobrevaloran las características que se corresponden con las expectativas del perceptor.

Según Bruner, hay dos tipos de determinantes en la percepción:

- Formales: las propiedades de las estimulaciones y del aparato receptor.

- Funcionales: las necesidades, emociones, actitudes, valores y experiencias del perceptor.

Hasta la década de los cuarenta, los psicólogos se ocuparon sobre todo de los determinantes formales de la percepción; en reacción a esa tendencia, los autores de la "new look" (Salomon Asch y otros autores que estudiaban la percepción social) empezaron a ocuparse de los determinantes funcionales. Para estos autores, además de los estímulos recibidos por los sentidos, hay otros factores que influyen en la percepción: las experiencias previas, las motivaciones, las defensas afectivas y las emociones del perceptor. Bruner trata de las variables intermediarias que se sitúan entre la experiencia previa y las necesidades del perceptor y su respuesta perceptiva: son las hipótesis del sujeto.

En un experimento realizado por Bruner y Goodman (1947) con niños de 10-11 años, la mitad de ello de familias pobres, y la otra mitad de familias ricas, los niños tenían que adecuar un círculo luminoso variable al tamaño de diferentes monedas. Según se pudo ver en los resultados, se sobrevaloraron los tamaños de las monedas más valiosas, y se infravaloraron los tamaños de las monedas menos valiosas. Pero fue mayor la distorsión de los niños de familias pobres.

La percepción se asienta pues sobre la formulación de hipótesis y sobre la toma de decisiones, influyendo en ella las necesidades, valores y deseos del sujeto. Así, Bruner pudo observar que los sujetos interpretaban de modo diferente una imagen humana ambigua, expuesta a ellos durante un breve momento. Los que tenían fuertes valores religiosos, percibían a un hombre en actitud de rezar; los que compartían valores de la ética protestante, percibían a un hombre trabajando.

\section{LA REPRESENTACIÓN}


El sujeto codifica y clasifica los datos que le llegan del exterior, reduciéndolos a categorías de las que dispone para comprender el entorno. Estas clasificaciones y codificaciones son procesos intermediarios entre los estímulos y la conducta. Son clasificaciones y codificaciones que dependen de las necesidades, experiencias, expectativas y valores del sujeto.

Para Bruner, el comportamiento no es pues algo que depende únicamente y mecánicamente de un estímulo objetivo externo; el sujeto transforma la información que le llega por medio de tres sistemas de representación: la representación enactiva, la representación icónica y la representación simbólica.

En la representación enactiva (enactive representation) el sujeto representa los acontecimientos, los hechos y las experiencias por medio de la acción. Así, por ejemplo, aunque no pueda describir directamente un vehículo como la bicicleta, o aunque no tenga una imagen nítida de ella, puede andar sobre ella sin tropezar. Los contornos de los objetos relacionados con nuestras actividades quedan representados en nuestros músculos. Este tipo de representación está pues muy relacionado con las sensaciones cenestésicas y propioceptivas que tiene el sujeto al realizar las acciones. Es un tipo de representación muy manipulativo.

La representación icónica (iconic representation) es más evolucionada. Echa mano de la imaginación. Se vale de imágenes y esquemas espaciales más o menos complejos para representar el entorno. Según Bruner, es necesario haber adquirido un nivel determinado de destreza y práctica motrices, para que se desarrolle la imagen correspondiente. A partir de ese momento, será la imagen la que representará la serie de acciones de la conducta.

La representación simbólica (symbolic representation), va más allá de la acción y de la imaginación; se vale de los símbolos para representar el mundo. Esos símbolos son a menudo abstracciones, que no tienen porqué copiar la realidad. Por medio de esos símbolos, los hombres pueden hipotetizar sobre objetos nunca vistos.

Al tratar de examinar la influencia que tienen estos tipos de representación en la educación, Bruner constató que incluso las personas que han accedido a la etapa de la representación simbólica, se valen todavía a menudo de la representación enactiva e icónica, cuando van a aprender algo nuevo. En consecuencia, Bruner aconseja a los educadores que utilicen en las escuelas la representación por la acción y la representación icónica, cuando vayan a enseñar algo nuevo.

Cada niño puede utilizar el nivel de representación que le permita su nivel de desarrollo. Un niño que está estudiando "la relieve" en el área de Naturaleza, puede valerse de la representación enactiva para construir su representación, subiendo y bajando las faldas de una colina. Utilizará la representación icónica, si se vale de una maqueta de curvas de nivel. Puede también valerse de la representación simbólica, si ha adquirido un nivel de evolución suficiente y es capaz de interpretar las cotas de relieve.

En consecuencia, Bruner rechaza la tendencia a la introducción demasiado temprana y precoz del lenguaje formal; incluso cuando el alumno haya llegado al nivel simbólico. El aprendizaje significativo se logra mejor, si pasa por las tres etapas:

\section{LA CONSERVACIÓN}


En los experimentos sobre la conservación se puede ver el paso de la representación icónica a la representación simbólica. Bruner y Kenney (1966) mostraron un ejemplo del cambio de la representación icónica a la representación simbólica en niños de 5 a 7 años. Utilizaron 9 vasos de tres diámetros diferentes y de tres alturas diferentes. Tal como aparece en la Figura-1, los vasos se colocaban en una tabla de $3 \times 3$.

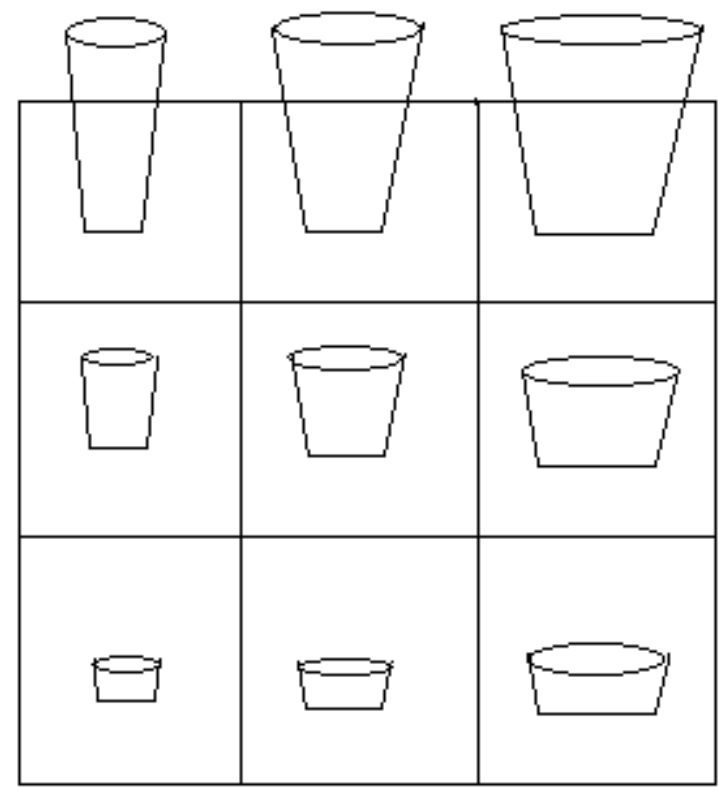

Figura1. Procedimiento de construcción de la matriz

(Bruner y Kenney, 1966)

Se le pedía al niño que mirara la tabla; acto seguido, el experimentador mezclaba los vasos y pedía al niño que hiciera algo parecido a lo que había antes. Después de haber logrado con éxito el primer ordenamiento, el experimentador mezclaba de nuevo los vasos, pero colocando el vaso bajo y estrecho, que al comienzo estaba en el extremo inferior-izquierdo, en el extremo inferior-derecho; acto seguido, se le pedía al niño que colocara los vasos como al comienzo, dejando en su sitio el vaso que el experimentador había cambiado de lugar.

En la tarea de reconstruir la matriz original, poniendo en su lugar los vasos desplazados de su lugar, no se constataron diferencias entre los niños de 5, 6 y 7 años. Casi todos los niños, ejecutaron bien el trabajo. La única diferencia estuvo en la mayor rapidez de los niños mayores al ejecutar el trabajo. En la tarea de construir la matriz, con la posición de un vaso cambiada, casi todos los niños de 7 años realizaron bien el trabajo. Ninguno de los más pequeños consiguió superar la tarea. En este experimento se pudo constatar que en los niños más pequeños influye mucho la imagen de la matriz original. Los niños de 5 años tienden a representar la matriz icónicamente, como si fuera una imagen espacial. Sin embargo, los niños de 7 años disponen ya de símbolos lingüísticos como la anchura o la altura.

Los niños que utilizan la representación icónica tienen una sensibilidad especial para la organización espacial e imaginaria de la experiencia, pero tienen menos sensibilidad para los principios y normas de ordenación de esa organización. Incluso el lenguaje que utilizan en la elaboración de la tarea no es un instrumento suficientemente trabajado para esa ordenación (Bruner, 1964). 
Según demostraron Piaget e Inhelder (1962), si se les presentan a niños de 4-7 años, dos recipientes iguales que tienen la misma cantidad de agua, en el momento en que se eche el agua de uno de esos vasos a otro más ancho o más estrecho, los niños dejarán de considerar que se trata de la misma cantidad de agua. Si el segundo vaso es más alto y estrecho, el niño dirá que hay más agua, porque llega a mayor altura; si es más ancho, dirá que hay menos agua, porque el nivel queda más bajo. Si cambia pues la forma del vaso, el niño no será ya capaz de conservar el volumen del líquido.

Las pruebas de conservación de Frank que menciona Bruner, tienen por objetivo evaluar esa capacidad en los niños. Evaluó en un experimento la capacidad de conservación de niños entre 4 y 7 años. Presentaba dos vasos normales e iguales, con la misma cantidad de agua, de forma que los niños pudieran ver que tenían la misma cantidad de agua (Figura-2). Luego, ponía junto a los vasos normales, un vaso de la misma altura, pero más ancho. A continuación, el experimentador echaba el agua de un vaso normal al vaso más ancho, y preguntaba al niño si había la misma cantidad de agua en los dos vasos. La capacidad de representación del niño aumenta con la edad. Más tarde, el experimentador oculta los tres vasos detrás de una pantalla, dejando ver sólo el borde superior de los vasos, de forma que no se pudiera ver el nivel del líquido. A continuación, se le preguntaba al niño a ver qué vaso tenía más agua, o si los dos vasos, el ancho y el normal tenían la misma cantidad de agua. Comparando con la prueba realizada sin pantalla, se dio un incremento extraordinario de juicios correctos en la prueba realizada con pantalla.

Figura 2

Pretest: los vasos A y B son del mismo tamaño y están llenos hasta el mismo nivel. Se vierte agua desde el recipiente $B$ al recipiente $C$ y se le pregunta al sujeto a ver dónde hay más agua, en $A$ o en $C$.

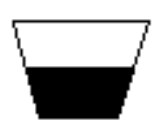

A
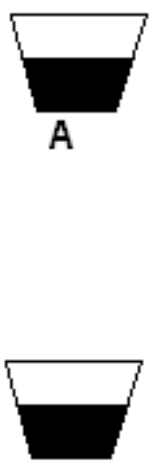

A

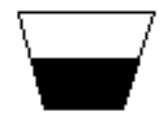

A

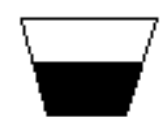

B
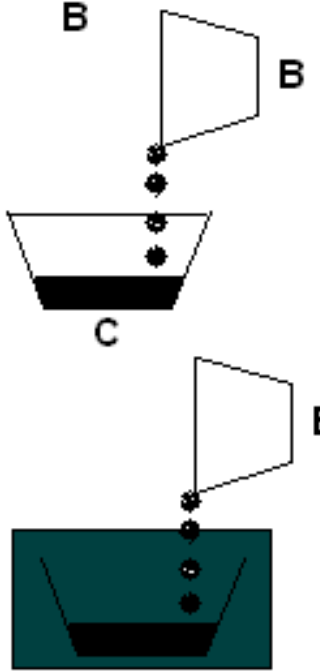

C

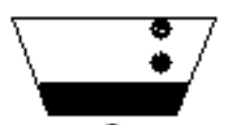

C

$B$

\section{Pretest}

Con pantalla

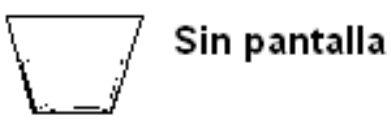

B 
El porcentaje de respuestas correctas pasó de 0 al $50 \%$ en los niños de 4 años; del $20 \%$ al $90 \%$ en los niños de 5 años; y del $50 \%$ al $100 \%$ en los niños de 6 años. Al ocultar los vasos detrás de la pantalla, los niños justificaban del siguiente modo sus juicios correctos: "es la misma agua" o "lo único que has hecho es pasar el agua de un vaso a otro".

Cuando se retiraba la pantalla, todos los niños de 4 años cambiaron de opinión. Confundidos por la información icónica, pensaban que el vaso ancho tenía menos agua. La mayor parte de los niños de 5 años mantenían sin embargo su juicio inicial, haciendo mención, eso sí, a la diferencia entre la apariencia y la realidad. Todos los niños de 6 y 7 años mantuvieron su postura inicial. Posteriormente, pasados unos minutos, se hizo un post-test, utilizando un vaso alto y estrecho, juntamente con los vasos normales, pero sin pantalla. La experiencia anterior no influyó en los juicios de los niños de 4 años: en esta nueva tarea, ninguno de ellos mantuvo la idea de conservación; pero la idea de conservación de los niños de 5, 6 y 7 años aumentó mucho del pretest al post-test.

Bruner y Kenney (1966) hicieron también otro experimento, con la finalidad de examinar el paso de la representación icónica a la simbólica. Se pedía a niños de 5, 6 y 7 años que dijeran cuál de dos vasos estaba más lleno y cuál más vacío. El concepto de estar "lleno" era considerado como muy fértil por Bruner, ya que incluía dentro de sí la idea de la proporción entre el volumen del recipiente y el volumen de la sustancia contenida en él. A un niño que se guía por el tipo de representación icónica se le hace muy difícil considerar que un pequeño recipiente medio lleno (por ejemplo, una copita) y un gran recipiente medio lleno (por ejemplo, un tonel), están llenos en la misma medida, ya que el recipiente grande tiene mayores medidas, en lo que se refiere al volumen. Para poder dar un respuesta correcta, el niño debe hacer una operación simbólica, superando la apariencia icónica. En la Figura-3 aparecen los 11 vasos utilizados en el experimento.

Hay cuatro tipos de vasos: los del Tipo-I (4, 7a y $7 \mathrm{~b}$ ) están todos llenos hasta la mitad, pero tienen una capacidad diferente; los del Tipo-II (2, 7a y 7b) están llenos del todo, pero tienen una capacidad diferente; los del Tipo-III (3, 8a y 8b) tienen una capacidad diferente, y uno de ellos está medio lleno, mientras que el otro está totalmente Ileno; los del Tipo-IV, son dos recipientes de la misma capacidad: en el dibujo 1 los dos están medio llenos, y en el dibujo 5 no están igualmente llenos.

Los criterios que utilizaban los niños de 5 a 7 años para decidir si los vasos estaban o no estaban igual de llenos eran similares. Estos criterios no se basaban en la proporción, sino en impresiones sensoriales que recibían directamente de la observación. El recipiente que aparentemente tenía un mayor volumen de agua, lo consideraban más lleno, siendo el nivel de agua el que indicaba la medida de ese volumen. Si el nivel del líquido era el mismo en los dos vasos, entonces se tenía en cuenta la anchura del vaso; cuando la anchura y el nivel eran los mismos, se tomaba en cuenta la altura del recipiente.

\section{PROCEDIMIENTO DE PROPORCIÓN}




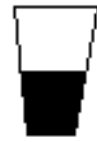

Dibujo 1

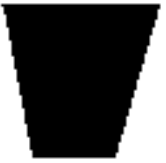

Dibujo 2

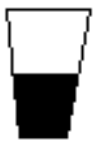

Dibujo 3

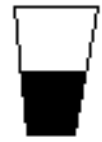

TipoIV

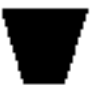

Tipo II

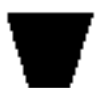

Tipo III

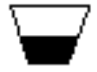

Dibujo 4

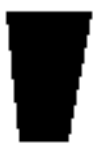

Dibujo 5
Tipo I

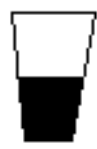

Tipo IV

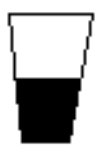

Dibujo 7a

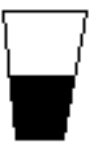

Dibujo $7 b$

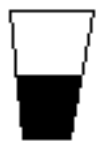

Dibujo 8a

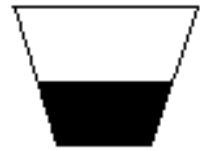

Dibujo 8b

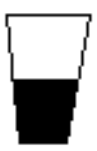

Dibujo9a

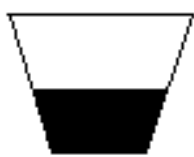

Dibujo 9b
Tipo III

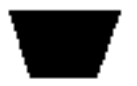

Tipo II

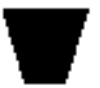

Tipo II
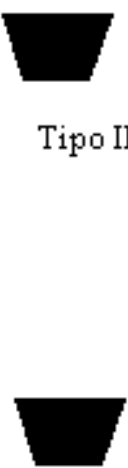

Tipo III

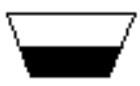

Tipo I

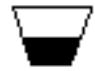

Tipo I

Figura 3. (Bruner eta Kenney, 1966)

En cuanto a cuál de los vasos se consideraba que estaba más vacío, si se tienen en cuenta los tres niveles de edad, se observa que los niños mayores tenía por costumbre comparar la porción llena y la porción vacía de cada vaso. El vaso "más vacío" era para ellos, el que tenía mayor volumen de espacio vacío aparente, y el "más lleno" era el que tenía el mayor volumen de espacio lleno. En consecuencia, llegaban a respuestas contradictorias, porque a la hora de decidir entre un vaso grande medio lleno y un vaso pequeño también medio lleno, el vaso que consideraban como el "más lleno" era el mismo que consideraban como el "más vacío". Sin embargo, los niños más pequeños, equiparaban el "estar vacío" con el "ser pequeño": el vaso que consideraban más vacío era el que aparentaba tener el menor volumen de líquido. Tomando los tres pares de vasos del TipoI, y teniendo en cuenta solamente los errores, para los niños de 7 años, el vaso más vacío era el que mayor espacio vacío tenía; sin embargo, para los niños de 5 años, el vaso más vacío era el que tenía menor volumen de agua. 
Según se puede deducir de estos experimentos, los errores de contradicción aumentan con la edad, teniendo mayor tendencia a considerar el mismo recipiente a la vez como más vacío y como más lleno. Mientras que los niños de 5 años se centraban únicamente en una dimensión (lleno-vacío), los niños de 7 años se fijaban en dos aspectos, aunque no tenían todavía medios para relacionar esos dos aspectos con un tercero, que sería el volumen del vaso. En opinión de Bruner, las dificultades se encuentran en pasar del formato icónico-perceptivo al formato simbólico. Cuando el niño llega a tener en cuenta el tercer aspecto, esto es, el volumen del recipiente, aparece el concepto simbólico de la proporción. Por medio de ese concepto, puede representar algo que no está perceptivamente presente. A los niños de mayor edad, a pesar de sus contradicciones, no les falta mucho para llegar al hallazgo.

Las investigaciones llevadas a cabo por Bruner, o mencionadas por él, marcan este sentido en la evolución cognitiva del niño: a la hora de clasificar hechos y objetos, en la medida en que van madurando, los niños utilizan cada vez menos los criterios perceptivos e icónicos, y utilizan cada vez más normas para organizar la realidad en estructuras jerárquicas supraordenadas. En la medida en que va adquiriendo las capacidades lingüísticas y en la medida en que las va actualizando en la organización de los hechos, va superando la organización perceptiva y empieza a organizar la realidad de acuerdo a normas más abstractas, basándose en principios de inclusión, exclusión etc.

Para superar el mundo perceptivo inmediato, es pues necesario traducir los acontecimientos del entorno a la forma simbólica de representación. El niño necesita un sistema que le permita trascender la situación presente, dándole la posibilidad de manejar algo que no pueda percibir directamente de la realidad. La representación icónica aparece ligada a las propiedades perceptivo-espaciales de los acontecimientos actuales. Es el lenguaje el que posibilitará el distanciamiento con respecto a la realidad inmediata, posibilitando hacer operaciones combinatorias y productivas con el objeto representado ausente. Este nuevo avance posibilitará al niño el que pueda diferir su gratificación.

\section{LA FORMACIÓN DE CONCEPTOS}

Cuando tratamos de clasificar los objetos, abstraemos algunas de sus cualidades y rechazamos otras. Para eso tenemos que representar los objetos, y para ello, tal como hemos visto, tenemos diferentes tipos de representación. La representación icónica, por ejemplo, es concreta y poco esquematizada; tiene grandes dificultades para liberarse de la configuración perceptiva concreta. Por eso, ese tipo de representación dificulta la adquisición de conceptos abstractos, basados en las características esenciales de los objetos.

¿Cómo llegan los niños a formar los conceptos? Los niños con predominio de representación enactiva, hacen clasificaciones basadas en el aspecto manipulativo; lo niños con predominio de representación icónica, hacen clasificaciones basadas en aspectos perceptivos. Para que lleguen a la clasificación lógica, tienen que traer al primer plano la forma de representación simbólica.

Oliver y Horsnsby (Bruner y cols., 1966) estudiaron estos puntos, presentando a sujetos de 6 a 19 años series de material figurativo y haciéndoles buscar sus características comunes. Se les presentaban dos conjuntos de palabra. Por una parte, plátano, melocotón, patata, carne, leche, agua, aire y bacilos; por otra parte, campara, bocina, teléfono, radio, periódico, libro, pintura y educación. En cada serie, los experimentadores empezaban señalando dos palabras (plátano-melocotón o campana-bocina) y se preguntaba 
al examinando sobre la similaridad existente entre los dos objetos. Al final de cada serie había una palabra que no combinaba bien con la serie. Los sujetos examinados debían de señalar la diferencia con respecto a los otros objetos.

Los autores encontraron diferencias notables en la forma de agrupación. Los niños de 6 años se guiaban por criterios de percepción: brma, tamaño, color, orientación, posición. Según iban avanzando en edad, daban más importancia a la

función del objeto. Hacia los 9 años, buscaban la función común de los objetos, sin tener en cuenta su utilización real.

Bruner, Goodnow y Austin estudiaron las estrategias de formación de conceptos en su libro A Study of Thinking. Mostraron a los estudiantes del experimento las 81 láminas. Las figuras de las láminas tenían cuatro dimensiones, que cambiaban en tres valores: forma (cruz, círculo, cuadrado), color (blanco, negro, con barras), número de márgenes (uno, dos, tres) y número de objetos (uno, dos, tres)..

Con esas imágenes se pueden formar conceptos, teniendo en cuenta que algunos estímulos serán ejemplos positivos del concepto, y otros estímulos serán ejemplos negativos del concepto.

El tipo de concepto más simple es el concepto de valor único, aquel que es definido por un solo atributo. Tiene en cuenta solamente el valor de una dimensión, dejando de lado las otras dimensiones. Por ejemplo, "todas las laminas de tres figuras". Cuando utilizamos una única característica común en la formación de conceptos, las posibilidades de agrupamiento son muy numerosas. Pero hay que tener en cuenta, que la mayor parte de los conceptos se definen por más de una característica. Bruner analiza las relaciones que se establecen entre estas características en los conceptos conjuntivos, disjuntivos y relacionales.

Para formar un concepto conjuntivo se necesitan de 2 a 4 atributos. El concepto conjuntivo lo definen dos (o más) características, y las dos son necesarias para describirlo. Por ejemplo: "todas las láminas de cruces rojas y de dos recuadros".

Par formar un concepto disjuntivo, se necesita una dimensión de entre dos o más dimensiones. Un valor de una dimensión u otro valor de otra dimensión. Los conceptos disjuntivos se definen por una u otra característica. Por ejemplo, "todas las láminas que tienen una cruz o una figura negra". En este caso, no son necesarias las dos características para que sea un ejemplo positivo del concepto. Es suficiente con el valor de una dimensión u otro valor de una dimensión diferente.

Los conceptos relacionales vienen definidos por las relaciones entre los valores, y no por la mera presencia de esos valores. Los atributos o dimensiones que los definen están relacionados entre sí. Por ejemplo, "todas las láminas que tienen la misma cantidad de figuras y márgenes".

La característica común que une a los diferentes tipos de conceptos es la de tener una estructura de clase lógica. Con esto se quiere decir que, una vez conocido el concepto, podemos decir sin ningún tipo de duda si un estímulo es o no es un ejemplo positivo de ese concepto.

Bruner, Goodnow y Austin (1956) utilizaron dos procedimientos diferentes para el estudio de la adquisición de los conceptos: el método de recepción y el método de selección. 
En el método de recepción se le explica al sujeto el tipo de concepto y el experimentador le presenta una de las 81 láminas, diciéndole que es un ejemplo positivo del concepto que él tiene en mente y que el sujeto tiene que adivinar. Luego, ocultando el estímulo inicial, el experimentador le presenta otro estímulo, y el sujeto tiene que decir si considera que es un ejemplo positivo o negativo del concepto, y también tiene que decir cuál es su hipótesis con respecto al concepto que el experimentador tiene en mente. El experimentador le debe de informar sobre si el pronóstico que ha hecho sobre el estímulo es o no correcto. El sujeto debe de continuar hasta dar con el concepto.

En el método de selección, es el sujeto mismo el que elige las láminas. Como en el experimento por recepción, también aquí, el experimentador le dice que tiene en mente un concepto, le presenta un estímulo que es una caso positivo de ese concepto, y le dice al sujeto que tiene que adivinar de qué concepto se trata. A partir de ahí, es el mismo sujeto quien selecciona los estímulos, uno a uno y en el orden que quiera, le dice al experimentador si se trata de un caso positivo o negativo del concepto y le pregunta si está o no en lo cierto. La tarea sigue hasta dar con el concepto correcto.

Observando en esas condiciones la resolución del problema de formación de los conceptos, Bruner y sus colaboradores se dieron cuenta de que los sujetos utilizaban diferentes estrategias.

Centrándose en el método de recepción, distinguen dos estrategias: la estrategia holistica y la estrategia parcial. En la estrategia holística, el sujeto toma como atributos definidores todos los valores del primer caso positivo. Partiendo de esa hipótesis, va rechazando los valores que no aparecen en los otros ejemplos positivos, hasta dar con el concepto correcto. En la estrategia por partes, el sujeto toma como hipótesis uno o algunos de los valores del primer caso positivo, y mantiene esa hipótesis, hasta que encuentra casos positivos o negativos que la falseen. En este caso, debe sustituirlo por una hipótesis que combine bien con casos pasados que guarda en su memoria.

La estrategia holística abre un proceso de verificación sistemática, rechazando progresivamente los atributos. En esta estrategia, serán únicamente los casos positivos los que den información significativa. En la estrategia por partes, surgen problemas a la hora de rechazar una hipótesis o de sustituirla por otra, ya que el sujeto debe recordar los casos pasados y encontrar una hipótesis que concuerde con ellos. Según pudieron constatar Bruner y colaboradores, los estudiantes universitarios utilizaban la estrategia holística, y los que se valían de esa estrategia identificaban más rápidamente el concepto que los que utilizaban la estrategia parcial. Esa diferencia era aún mayor, en la medida en que crecía la dificultad de la tarea.

Bruner y colaboradores, identificaron también dos estrategias cuando utilizaron el método de selección: la estrategia focalizada y la estrategia de verificación sucesiva de hipótesis.

Al utilizar la estrategia focalizada, coge como atributos que definen al concepto todos los valores del primer caso positivo. El primer caso positivo funciona como foco en el proceso de verificación. Partiendo de esa hipótesis, irá seleccionando las láminas que le ayudarán a desechar atributos. Se puede utilizar un enfoque conservador o uno arriesgado. En el enfoque conservador, se elegirán los valores que se diferencien en un solo atributo del foco; en el enfoque arriesgado, los valores seleccionados se distinguirán en más de un atributo del foco. Si el ejemplo es positivo, el proceso de rechace será rápido. Si es negativo, dará poca información. 
En la estrategia de verificación sucesiva de hipótesis, el sujeto toma como hipótesis una o varias características del primer caso positivo. Algunas veces, hará un análisis simultáneo de todas las hipótesis posibles, rechazando, después de cada caso, las que no se tienen en pie. Otra veces, hará un estudio sucesivo de la hipótesis, tomándolos uno a uno. El sujeto comienza por una hipótesis y la mantiene, si predice bien la clase del ejemplo. En el caso contrario, la cambia por otra que concuerde con toda la experiencia pasada.

Tal como sucedía en el método de recepción, las estrategias focalizadas fueron más eficaces que la de verificación sucesiva, y los estudiantes analizados por Bruner y colaboradores las utilizaban más frecuentemente. En la estrategia de verificación sucesiva de hipótesis, el sujeto limita su elección a las láminas que le permiten comprobar directamente su hipótesis. Una vez hecha la elección, pasa a una nueva hipótesis, y la comprueba de nuevo directamente. Esta estrategia exige recordar los ejemplos comprobados de antemano. Esta estrategia exige pues más a la memoria que la estrategia focal. Además, la estrategia de verificación sucesiva dificulta saber cuáles son los criterios que no tienen relevancia.

Según pudieron comprobar Bruner, Goodnow y Austin, los conceptos disjuntivos eran más difíciles de adquirir que los conceptos conjuntivos. En el caso de los conceptos disjuntivos, la información negativa tiene mas relevancia que la información positiva; para rechazar las hipótesis, se necesitan ejemplos negativos del concepto. Y como los sujetos muestran preferencia por la información positiva, los conceptos disjuntivos son más difíciles de adquirir. Si se mira a la evolución del niño, antes de diez años el niño tendrá en cuenta solamente los ejemplos positivos en la formación de conceptos. Más tarde, irá teniendo en cuenta sistemáticamente la información negativa.

De todas formas, Bruner admite que él trabajo sobre todo con conceptos artificiales. Más tarde, incluyeron en la investigación elementos y tareas de mayor realismo. En los trabajos abstractos de los conceptos artificiales les era bastante indiferente a los sujetos el que fuera uno u otro el atributo significativo; pero, desde el momento en que se introdujeron temas realistas de personas como material de formación de conceptos, los sujetos mostraron preferencias por ciertas hipótesis. Veían cada estímulo como una historia. Les resultaba mucho más difícil la falsación de hipótesis temáticas, ya que en los conceptos temáticos no se trata de una mera lista de atributos, sino que entraba en juego un sentido y un significado.

\section{LA CODIFICACIÓN}

Cuando vamos más allá de la información dada, es porque disponemos de un sistema de codificación más extenso; una vez en posesión de ese sistema de codificación, podremos lograr una sobreinformación, basados en probabilidades contingentes que hemos aprendido o en principios aprendidos para relacionar el material. Una parte importante de la transferencia del aprendizaje consiste justamente en aplicar sistemas de codificación aprendidos a nuevos sucesos.

Pero, ¿qué condiciones tienen que darse para que el sujeto pueda aplicar sus aprendizajes a nuevas situaciones?. Primeramente, hay que mencionar el papel de las disposiciones. Para ello, Bruner saca a relucir el clásico estudio de Hull. Los sujetos deben de memorizar qué sílabas sin sentido se relacionan con tal o cual figura. Un subconjunto de la serie de figuras lleva una etiqueta, y el otro subconjunto lleva otro. Sin que el sujeto lo sepa, esos subconjuntos tienen una propiedad que los define. Al sujeto se le asigna la tarea de 
adivinar la etiqueta que corresponde a cada dibujo. Si el sujeto piensa que el trabajo consiste en la memorización de etiquetas, esa predisposición le impide una más rápida adquisición de los conceptos, y no le permite recordar bien; si se le explica claramente al sujeto la finalidad real del experimento, es decir, si se le dice que el objetivo es llegar a saber qué es lo que hace que unos dibujos lleven una etiqueta y otros lleven otra, en esa situación adquieren más fácilmente el concepto. La disposición preinducida puede llevarle a un comportamiento más mecánico o a utilizar sistemas apropiados para una codificación más genérica de hechos y principios.

El máximo proveedor de instrucciones inductoras es la historia profesional o social de cada uno. Por deformación profesional, echamos mano de códigos formales comunes a nuestra experiencia o profesión, a la hora de codificar la realidad. Llegado a este punto, Bruner nos recuerda las disposiciones típicas de la persona, que le llevan a tomar una actitud más o menos abstracta o concreta ante las situaciones de resolución de problemas. Las personas que puntúan alto en concreción, tratan y procesan los hechos desde la perspectiva de su identidad particular, sin trascender a lo más general. Las personas que tienen una actitud más abstracta, trascienden lo particular y lo engloban dentro de categorías más generales, como un caso particular de ellas.

La generalidad de los sistemas de codificación que utilizamos para ir más allá de los dado, depende también de estar suficientemente motivado. En opinión de Bruner, los que tienen un nivel de motivación demasiado bajo o demasiado alto, generan una actividad cognitiva orientada más bien a la concreción. Para que se genere una tendencia a la adquisición de un sistema de codificación generalizable, se requiere un nivel de motivación mediano.

También el nivel de adiestramiento tiene que ver con la generalización de la codificación. Cuanto más entrenado esté el sujeto, llegará a una mayor generalización de la codificación, siempre que la motivación no sea demasiado grande o demasiado pequeña. Para que un aprendizaje se pueda generalizar, es necesario que se posibilite el descubrimiento de regularidades de nivel inferior, así como la combinación de esas regularidades en sistemas superiores de codificación.

En lo referente a la diversidad de situaciones entrenamiento, si queremos generalizar de una situación a situaciones similares, tenemos que abstraer las propiedades definitorias de la clase de hechos correspondientes a la situación original. Si queremos que el sujeto transfiera el aprendizaje de una situación a otra, tendremos que mantener por más tiempo a los sujetos experimentales y habrá que exponerles a más tareas originales.

Tratando de la invención de sistemas de codificación, hay que mencionar los cambios habidos en el último siglo. Si las teorías de la física newtoniana eran presentadas como descripciones de los descubrimientos sobre la naturaleza, y no como un sistema de constructos teóricos, la ciencia actual construye teorías y modelos formales con capacidad de predicción. El Universo no es una realidad objetiva, sino un conjunto de constructos que inventan los científicos para comprender y predecir sus observaciones. Los sistemas teóricos aparecen como sistemas de codificación que posibilitarán al científico generalizar más allá de los datos observados. 
La teoría o el modelo es un sistema de codificación, vacío de contenidos particulares. La producción de un sistema de codificación generalizable, consiste justamente en esta operación de vaciado del contenido concreto. El aprendizaje de lo general es la "cara" de la moneda. La "cruz" de la moneda consiste en la operación de vaciado o de abstracción.

Bruner aconseja a los estudiantes que aprendan a utilizar estos sistemas de codificación, porque eso les posibilitará ir más allá de lo dado. Los estudiantes deben de adquirir sistemas de codificación que sean aplicables en situaciones distintas a las originales.

\section{EDUCACIÓN Y DESARROLLO}

La educación es una invención de la sociedad. En las sociedades primitivas, el niño aprende imitando modelos directos del mundo adulto que le rodea desde el nacimiento. En las sociedades complejas, los niños no toman parte directa en el mundo de los adultos; aprenderán de los adultos, pero en instituciones fuera del contexto natural.

El uso que el hombre vaya a hacer de su inteligencia, depende de su capacidad para inventar y utilizar herramientas y tecnologías; éstas le permiten maximizar sus capacidades. Según Bruner, el lenguaje es el mejor ejemplo de una tecnología potente, ya que se utiliza no sólo para comunicarse, sino también para representar, codificar y transformar la realidad.

Para Bruner, una teoría del desarrollo intelectual tiene que ayudarnos a comprender mejor cómo tiene que ser la educación. Una teoría de la instrucción tiene que aclarar los criterios que tienen que tenerse en cuenta para organizar un clima de estudio favorable para un aprendizaje adecuado. Tiene que establecer las normas para lograr de modo eficaz los resultados que se quieren obtener. Habría que distinguir entre una teoría de la instrucción que sería normativa, de una teoría del aprendizaje que sería descriptiva. La teoría del aprendizaje nos dice qué ocurre; la teoría de la instrucción nos dice qué hay que hacer para que el aprendizaje sea eficaz.

Para Bruner, una teoría de la instrucción tienen que cumplir las siguientes condiciones:

- Tiene que tomar en consideración factores personales del alumno como. Más el interés, la curiosidad, el placer, el deseo de aprender.

- Los temas de estudio hay que adecuarlos al nivel del alumno. Según Bruner, se puede enseñar cualquier tema a una persona de cualquier edad, siempre que se adecuen al nivel de desarrollo del alumno.

- El profesor debe conseguir que el alumno sea consciente de la importancia de sus esfuerzos para conseguir sus objetivos a largo plazo. Para Bruner, el conocimiento de los resultados debe de ocurrir en el momento en que está resolviendo el problema. El refuerzo es, en este sentido, la retroalimentación que proviene de los resultados del ensayo. Si ese refuerzo llega demasiado tarde o demasiado temprano, no se aprovecha.

- El aprendizaje por descubrimiento es el único tipo de aprendizaje que puede infundirle confianza en sí mismo. Este tipo de aprendizaje libera al alumno del control de la motivación externa. 
- Si la escuela ha de llevar a cabo una labor educativa adecuada y equilibrada, debe de permitir al alumno que se comporte de un modo intuitivo, cuando así lo requiera. El pensamiento intuitivo permite enseñar al niño la estructura fundamental de un tema, antes de que sea capaz para el razonamiento analítico. Es el tipo de conocimiento que se consigue por medio del arte y de la poesía.

\section{EL LENGUAJE Y LA CULTURA}

Bruner piensa que el niño tiene algún tipo de conocimiento del mundo real, aun antes de ser capaz de clarificar con eficacia los misterios de la sintaxis. Antes de aprender a hablar, el niño dispone de unas capacidades cognitivas:

- Muchos procesos cognitivos de la infancia van orientados a actividades dirigidas a unos objetivos.

- Los niños son sociables en el sentido de que están predispuestos a responder a la voz, al rostro, a los gestos y a las acciones de los que le rodean. Los adultos activan muy precozmente las estructuras de interacción de los niños. Los dispositivos interaccionales de los niños, a su vez, engloban rápidamente las acciones de los adultos. El dispositivo primordial que tienen los niños para lograr sus objetivos es un miembro adulto de su propia familia.

- Muchas de las primeras acciones de los niños se dan en situaciones restringidas de la familia, y muestran un nivel de sistematización muy elevado. Dice Bruner, que cuando el campo de acción del niño está delimitado, lo que ocurre en ese campo es tan ordenado y sistemático como lo que ocurre en la vida de un adulto. Cuando el niño entra en el mundo del lenguaje y de la cultura, está ya preparado y capacitado para descubrir e inventar formas sistemáticas de relacionarse con las exigencias de la sociedad y con las formas lingüísticas. La comunicación prelingüística y la lingüística suceden en un campo restringido: en esas situaciones, el niño y el educador combinan todos los elementos para abrir una comunicación eficaz.

- Según Bruner, el carácter sistemático de las capacidades originales del niño es extraordinariamente abstracto. Parece como si los niños se rigieran por normas, a la hora de entablar sus relaciones espaciales, temporales o de causa-efecto. Estas capacidades cognitivas son los mecanismos básicos para la adquisición del lenguaje. Para pasar de una comunicación prelingüística a una comunicación lingüística, es necesario un escenario rutinario y familiar, que posibilite el que el niño pueda comprender lo que está sucediendo. Son estas rutinas las que Bruner llama Sistemas de Apoyo a la Adquisición del Lenguage (LASS: Language Acquisition Support System).

EI LASS asegura el paso de la comunicación prelingüística a la comunicación lingüística de estas cuatro formas:

- Bruner dice, mencionando a Bickerton (1981), que los niños están "bioprogramados" para percibir unas distinciones entre los acontecimientos del mundo real, comunicarse respecto de ellas y hacer las distinciones lingüísticas correspondientes. En la medida en que la sincronización de la interacción entre el adulto y el niño se concentra en estas distinciones, ayudamos al niño a que 
pase de su expresión conceptual a una representación lingüística apropiada. Esta distinciones las encontraremos en los formatos.

- El adulto ayuda al niño modelando frases que sustituyan a la comunicación gestual y vocal primitiva del niño, para que pueda cumplir las funciones comunicativas pertinentes.

- Lo que caracteriza al formato de los juegos es que está constituido por "hechos" generados y recreados por medio del lenguaje. El formato de los juegos ofrecerá una amplia gama de oportunidades para aprender el lenguaje y utilizarlo.

- Cuando la madre y el niño se integran en formatos de rutina, se ponen en marcha procesos psicológicos y lingüísticos que se generalizan de un formato a otro.

Los cuatro mecanismos mentales que hemos mencionado componen el equipamiento mental mínimo que el niño necesita para adquirir el lenguaje. Sin embargo, quien quiera aprender una lengua necesita algo más que esa maquinaria elemental. No basta con estar dotado de la capacidad léxico-gramatical, hace falta saber cómo utilizar el lenguaje en forma comunicativa.

¿Cómo consigue el niño actuar en los demás por medio del lenguaje? Es el Dispositivo de Apoyo a la Adquisición del Lenguaje (LASS) el que regula la interacción entre las personas, el que ayuda a la utilización correcta del lenguaje. Ese dispositivo no es puramente lingüístico. Es la característica central que tiene el sistema para la transmisión cultural; el lenguaje es creador de esa cultura, y , a la vez, instrumento de ella. En su interacción con el primer lenguaje, el niño dispone de la primera oportunidad para interpretar los textos culturales. Al aprender cómo decir, aprende lo que es canónico, obligatorio y valorado en y por la cultura. El niño aprende esto último por medio de un tipo de comunicación que no llega a ser lingüístico.

Para Bruner, el medio principal del LASS es el "formato". El formato es una pauta estandarizada de interacción entre un adulto y un niño, una pauta que tiene funciones delimitadas que son reversibles. Incluye no solamente la acción, sino incluso la comunicación que organiza, dirige y completa esa acción. Más tarde, los formatos se organizan en grupos y serán considerados como módulos que construyen una interacción social y un discurso más complejo. En la medida en que la abstracción va creciendo, los formatos se liberan de los esquemas específicos y pueden ser utilizados en situaciones diferentes. La forma va diferenciándose del contexto.

El formato es una interacción contingente: la respuesta de cada miembro depende de la contestación previa del otro. Los formatos crecen, y pueden hacerlo hasta convertirse en suficientemente complejos. Cuando se trata de la consecución de objetivos, los formatos pueden incorporar otros medios y otras estrategias, incluidos los simbólicos o lingüísticos.

El formato integra las intenciones comunicativas del niño en una matriz cultural. Asocia la acción que se ejerce sobre un objeto con la acción que se ejerce sobre el otro sujeto. Las acciones indefinidas del recién nacido irán especificándose por la intermediación del otro. El funcionamiento de esta interacción social les lleva a repeticiones, pero no a repeticiones mecánicas y estereotipadas, sino a repeticiones de las que surgen cambios imprevistos. Sea como sea, el niño debe disponer de un conjunto complejo de savoir-faire, que sea transferible y creativo, y que abarque desde lo perceptual o motriz, hasta lo conceptual, social o lingüístico. 
Por medio de la coordinación de esos savoir-faire se lograrán realizaciones que puedan describirse por normas gramaticales.

En cuanto a la continuidad entre la comunicación prelingüística y la comunicación lingüística, Bruner subraya los siguientes puntos:

- La correspondencia entre los conceptos sobre el mundo real y las formas gramaticales: es el problema de la relación entre la semántica y la sintaxis que Bruner no ha estudiado directamente.

- La continuidad entre las funciones de la comunicación prelingüística y la comunicación lingüística, que ofrece una importante plataforma para el desarrollo de los procedimientos referenciales y de petición. La continuidad de la función permite un progreso por sustitución. En relación concretamente con la referencia, la madre mantiene con el niño durante meses una pauta de interacción constante sobre la denominación de los objetos. La estabilidad del formato hace que la función se mantenga constante. Cuando el niño adquiere formas nuevas, las utiliza para cumplir las funciones del viejo formato. Cuando no es capaz de utilizar las formas nuevas, vuelve a las antiguas. La forma nueva puede transformar o extender la función antigua, pero existe continuidad. La continuidad funcional proporciona una base al adulto para que pueda sintonizar adecuadamente con el niño.

- En lo que se refiere a la función constitutiva que cumple el lenguaje en el conocimiento del mundo real, la función de creación del mundo en el que vive el niño depende de los formatos de los juegos.

- Respecto a la similaridad de los procesos cognitivos, Bruner dice que los procesos conceptuales que dan continuidad al desarrollo, procesos comunes a la mente en general y al lenguaje en particular, son procesos sociales que se comparten en la comunicación prelingüística y lingüística.

La adquisición temprana del lenguaje depende de la utilización del contexto en la creación e interpretación de mensajes por parte de la madre y por parte del niño. Una comunicación temprana adecuada depende de un contexto familiar compartido; en ese contexto los interlocutores pueden manifestar claramente sus intenciones comunicativas. Pero, para Bruner, tanto los niños como los adultos seleccionan y construyen el contexto. Los contextos que se crean deben ser manejables, convencionales, de forma que faciliten manifestar al interlocutor lo que tenemos en mente. Los formatos son versiones especializadas del contexto. Los primeros formatos están prefabricados: los interlocutores no tienen necesidad de dar pistas sobre el contexto, porque tratan de interacciones habituales como la comida, los juguetes etc. Cuando se extiende el campo, se opta por la convencionalización. Los contextos naturales se estructuran en formas convencionales y se ordenan como formatos.

\section{BIBLIOGRAFIA}

BICKERTON, N. (1975). Reflections of Language. New York: Random House.

BOWER, T. G. R. (1967). The development of object permanence: some studies of existence constancy. Perception and Psychophysics, 2, 411-418. 
BRown, R. W. (1956). Language and categories. Appendix in J.S. Bruner, Jacqueline J. Goodnow, \& G.A. Austin, A study of thinking. New York: Wiley.

BRUNER, J. S. (1951). Personality Dynamics and the process of perceiving, in R.R.Blake y G. Ramsey (comp.). Perception, an approach to personality. New York: Ronald.

BRUNER,J. S. (1961). The act of discovery. Harvard Educational Review, 31, 21-32.

BRUNER, J. S. (1964). The course of cognitive growth, American Psychologist, 19, 1-15.

BRUNER, J. S. (1966). Studies in cognitive growth. New York: John Wiley.

BRUNER, J. S. (1971). The Relevance of Education. New York, W. W. Norton and C. Inc. Traducción española: La importancia de la educación. Barcelona: Paidos. 1987.

BRUNER, J. S. (1983). Child's Talk. Learning to Use Language. New York, W.W. Norton. Traducción española: El habla del niño. Barcelona: Paidós. 1986.

BRUNER, J. S. (1983). Le développement de l'enfant: savoir faire, savoir dire. Paris: Presses Universitaires de France.

BRUNER, J. S. (1984). Acción, pensamiento y lenguaje. Madrid: Alianza Editorial

BRUNER, J. S. (1986). Actual Minds, Possible Worlds. Cambridge: Harvard University Press. Traducción española: Realidad mental y mundos posibles. Barcelona: Gedisa, 1988.

BRUNER, J. S. (1988). Desarrollo cognitivo y educación. Madrid: Morata.

BRUNER, J. S. (1990). Acts of Meaning. Cambridge: Harvard University Press.

BRUNER, J. S. (2002). Pourquoi nous racontons-nous des histoires?. Paris: Editions RETZ.

BRUNER, J. S. y GOODMAN C. C. (1947). Value as need as organizing factors in perception, Journal of Abnormal and Social Psychology, 43, 33-44.

BRUNER, J. S., GoOdNow, J. J. y AUSTIN, G. A. (1956). A Study of Thinking. New York: Wiley. Traducción española: El proceso mental en el aprendizaje. Madrid: Morata.

BRUNER, J. S. y HASTE, H. (1987). Making sense. The Child's construction of the World. New York: Methuen. Traducción española: La elaboración del sentido. La construcción del mundo por el niño. Barcelona: Paidos. 1990.

BRUNER, J. S. y KENNEY, H. (1966). The development of the concepts of order and proportion in children. In J.S. Bruner, R.R. Olver, P.M. Greenfield et al. (Ed.), Studies in Cognitive Growth. New York: Wiley.

BRUNER, J.S., MANDLER, J., O'DOWD, D. y WALLACH,M.A. (1958). The role of overlearning and drive level in reversal learning. Journal of Comparative and Physiological Psychology, 51(5), 607-613.

Bruner, J. S., Olver, R.R., Greenfield,P.M. et al. (1966). Studies in cognitive growth. New York: Wiley.

BRUNER, J. S., POSTMANL. y RODRIGUES,J. (1951). Expectation and the perception of colour. American Journal of Psychology, 64, 216-227. 
BUTTHERWORTH, G. (1987). Algunas ventajas del egocentrismo. In J.S. Bruner y H. Haste (comp.), Making sense. The Child's construction of the World. New York: Methuen.

DONALDSON, M. (1978). Children's Minds. New York: Norton.

DUNN,J. (1987). La comprensión de los sentimientos: las primeras etapas. In J.S. Bruner y H. Haste (comp.), Making sense. The Child's construction of the World. New York: Methuen.

DONALDSON, M. (1978). Children's Minds. New York: Norton.

FRANK, F. (1966). Perception and language in conservation. In J. S. Bruner, R.R. Olver, P.M. Greenfield et al. (Ed.), Estudies in cognitive growth. New York: Wiley.

GolDSTEIN, K. (1939). The organism. New York: American Book Co.

LASHLEY, K. S. (1938). Experimental analysis in instinctive behavi or. Psychological Review, 45, 445-472.

LASHLEY, K. S. (1938). The problem of serial order in behavior. In L.A. Jeffress (Ed.), Cerebral mechanisms in behavior: The Hixon Symposium. New York: John Wiley.

LIGHT, P. (1987). La adopción de roles. In J.S. Bruner y H. Haste (comp.), Making sense. The Child's Construction of the World. New York: Methuen.

MANDLER, G. (1962). From association to structure. Psychological Review, 69, 415-427.

MAYER, R. E. (1983). Thinking, Problem Solving, Cognition. New York: W.H. Freeman and Company.

NAIR, P. (1963). An experiment in conservation. In Center for Cognitive Studies Annual Report, Harward University.

OLVER, R. R. (1961). A developmental study of cognitive equivalence. Tesis doctoral. Radcliffe College.

PIAGET, J. (1964). Six études de psychologie. Ginebra: Ed. Gonthier.

PIAGET, J. y INHELDER, B. (1962). Le développement des quantités physiques chez l'enfant. Neuchâtel, Suiza: Delachaux \& Niestlé.

Postmañ. y Bruner, J. S. (1948). Perception under stress. Psychological Review, 55, 314-323.

REED, H. B. (1946). Factors influencing the learning and retention of concepts. I. The influence of set. Journal of Experimental Psychology, 46, 107-112.

RIGNEY,J.C. (1962). A developmental study of cognitive equivalence transformations and their use in the acquisition and processing of information. Tesis doctoral. Dadcliffe College, Department odf Social Relations.

SCAIFE, M. y BRUNER, J. S. (1975). The capacity for joint visual attention in the infant. Nature, 253, 265-266. 


\title{
Contactar
}

Revista lberoamericana de Educación

\author{
Principal OEI
}

